\title{
AN IMPROVED METHOD FOR THE PRODUCTION OF ANTIMENINGOCOCCIC AND OTHER SERUMS
}

\author{
JOHN F. ANDERSON \\ From the Research Laboratories E. R. Squibbs and Sons, New Brunsterick, N, J.
}

Most of those who have had to do with the preparation of antibacterial serum have experienced the long time required for immunization and loss of animals usually associated with the preparation of such serums. The usual period assumed to be required for the immunization of horses with meningococci and other bacteria was from 4 to 8 months until Flexner and Amoss ${ }^{1}$ published their notes on the rapid production of antidysenteric serum based on the earlier work of Fornet and Müller, ${ }^{2}$ Bonhoff and Tsuzuki ${ }^{3}$ and especially Gay ${ }^{4}$ and his colleagues, in which it was shown that by the repeated injection of antigenic materials at short intervals a quicker and more exalted response in antibody formation was produced. The method used by these various workers consisted in its essential details in the injection of the antigenic substances usually for 3 successive days with a variable period of resting. Previous to the application of these earlier results by Flexner and Amoss to production of serum on a large scale, it had been the usual practice in the production of antibacterial serum to inject horses with cultures of bacteria of different periods of growth and manner of culture, oftentimes containing autolysates or soluble bacterial products. The injections were given by various routes-subcutaneously, intramuscularly and intravenously -but the frequency of severe reactions, when the intravenous method was used, greatly lessened the employment of that method. Flexner and Amoss immunized their horses for the production of antidysenteric serum by 3 successive intravenous injections of living cultures or toxin with intervening rest periods of 7 days. They state that a suitable serum for therapeutic use in man could be prepared in about 10 weeks.

Received for publication Aug. 16, 1920.

T Jour. Exper. Med., 1915, 21, p. 515.

2 Ztschr. f. biol. Techn. u. Method., 1908, 1, p. 201.

R Ztschr. f. Immunitätsforsch., 4, p. 180. Tsuzuki, M., ibid., p. 194.

4 Gay and Fitzgerald: University of California Publications in Pathology, 1911-14, 2, 77 Gay, F. P., Ergebn. d. Immunitätsforsch, exper. Therap. Bakteriol. u, Hyg., 1914, 1, p. 231 Loke, E., University of Californiz Publications in Pathology, 1911-14, 11, 0. 91. 
Amoss and Wollstein ${ }^{5}$ applied the same methods to the production of antimeningococcic serum, but quickly encountered the difficulties of other workers in the severe and even fatal reactions of their horses. They endeavored to overcome this drawback by the use of the so-called desensitizing injection as described by Briot and Dopter. ${ }^{6}$

As used by Amoss and Wollstein, the desensitizing injection, consisting of $1 / 20$ to $1 / 10$ of a 24 -hour agar slant culture was administered intravenously 2 hours before the full dose was given. They state that by the use of the desensitizing injection the severe reactions are avoided and that a satisfactory serum may be produced in 8 to 12 weeks instead of the much longer period required by other methods of immunization.

We had been using for some time the intravenous method injection but had been much troubled by the severe and even fatal reactions, so that when the work of Amoss and Wollstein appeared, the methods advised by them were given a trial.

It has been found that the time required for immunization was reduced by the method of 3 successive intravenous injections with a resting period of 7 days, but that the severe and even fatal reactions in the horses were not prevented by the use of a desensitizing dose. It was also found that it was not a satisfactory procedure to attempt to regulate the dose by the temperature curve as they suggested when many horses were under treatment.

As the demands for antimeningitic serum by the military forces were great and insistent, it was quickly realized that some methòd must be devised by which valuable animals producing a high titer serum could be prevented from dying and that the period required for immunization could be reduced even below that stated by Amoss and Wollstein.

Severe reactions were more frequently encountered among the horses used in the production of antimeningococcic serum than those used for the production of antipneumococcic serum, and the time required for immunization was appreciably longer, but severe and even fatal reactions occurred also in antipneumococcic horses.

As there were a considerable number of horses already producing serum of a high titer immunized by various procedures, it appeared more important to determine immediately, if possible, means by which severe reactions in these animals could be prevented.

5 Jour. Exper. Med., 1916, 23, p. 403.

- Compt. rend. Soc. de biol., 1910, 69, p. 174. 
Accordingly various modifications were made in the manner of growing the meningococci, preparing the emulsion for injection and method of injection. Any one who has witnessed the beginning, development and termination of a severe or fatal reaction in a horse following the injection of meningococci will have been struck by the similarity of the symptoms to those of anaphylaxis in the smaller animals, such as the guinea-pig, and it was not an unnatural assumption that the reaction in the horse was a manifestation of anaphylaxis or hypersensitiveness to the meningococci or its products, especially as the reaction is more frequently encountered later than the third or fourth course of injection, according to the Amoss-Wollstein scheme, or after the third or fourth week of treatment by other plans of immunization. This is, however, not invariably true as our records show a number of animals that had severe reactions, even followed by death, in some instances on the first day of treatment and a larger number during the first week of treatment.

Before going further with the discussion of the cause of the reaction and methods for prevention, I shall give a brief description of the reaction.

The reaction generally appears within 10 minutes and often in less than 5 minutes after an intravenous injection of meningococci or other bacteria. The first symptoms are rapid and shallow breathing, restlessness, sweating and general weakness.. The heart beat is increased but not in proportion to the respiratory difficulties. In the milder type of reaction the respiratory rate and character gradually improve and in 6 or 8 hours the animal returns to normal. In the severer reactions, the symptoms mentioned become aggravated, especially the difficulties of breathing and general weakness, so that the animal makes violent efforts to take in sufficient oxygen by the employment of all the accessory respiratory muscles. The horse finally becomes so weak as not to be able to stand and either falls or lies down, and is unable to rise. The temperature remains about normal. When death occurs it is apparently due to respiratory failure. Postmortem shows nothing distinctive; the right heart is full; the lungs are not distended or emphysematous; but the abdominal blood vessels appear distended with blood. Death has occurred following the first injection given, and in a number of instances has taken place within 5 minutes of the injection. If the symptoms do not appear within an hour after the injection, no reaction will 
follow. In our experience with about 100 horses observed for the reaction, it has had no relation to the number of the injection in a series, the series of injection, the rapidity with which the injection was made or whether the injection consisted of killed or live meningococci, but only when the injection was given intravenously.

In addition to the general reaction described, most horses show an elevation of temperature running from 1 to $3 \mathrm{C}$. after an intravenous injection of meningococci, and animals may show a rise of as much as 3 degrees and no general reaction.

As stated, we made use of the desensitizing injection suggested by Dopter, but early found that by no modification of its use could we surely prevent the development of general and fatal reactions. We then began to question whether the reactions were a manifestation of hypersensitiveness to the meningococci or their products and in a short time became satisfied that such was not the case; but that the symptoms were due to the action of a poison present in the injected material. That this is true in some instances at least is shown by the development of severe reactions following the first injection ever given the animal, or during the first series of injections. Furthermore, the theory was supported by the fact that in the reactions could be prevented by the procedure, which I shall describe, and by the production of a similar reaction in rabbits after a single and first intravenous injection. The latter part of the work will be reported in detail in a subsequent paper.

As soon as it was realized that the reactions were caused by the action of some toxic substance in the material injected, efforts were directed to its removal and were early rewarded with success.

In going over each step in the preparation of antigen, we found that it was the custom, chiefly because of its convenience, for the bacteriologic laboratory to prepare the antigen for the day's injection as early as possible, and that it was the custom for the veterinary department to make the injections at a time most convenient for the other work to be done. In this way the injections usually were made within an hour after the preparation of the antigen, but that in some instances several hours elapsed. It was further found that reactions were more frequent and more severe when a considerable period had elapsed between the making of the antigen and the injection.

All injections of meningococci and other bacterial antigens were now made as soon after their preparation as possible, in no case 
longer than 30 minutes and ustally not more than 10 minutes after preparation. This reduced the frequency of the reactions, but did not entirely eliminate them. At this point some work under way on rabbits gave us a valuable lead. If the antigen were prepared in the usual way and injected intravenously into rabbits, the rabbit frequently died within a short period, but if the suspension of bacteria were centrifuged, washed once and resuspended, the injection of this washed suspension of bacteria would be without ill effect on the rabbit.

Accordingly, the antigen for the horses was prepared in a similar way and it was soon found that the use of a washed suspension of meningococci, given immediately after preparation, resulted in the elimination of all severe reactions, and of practically all general reactions except an occasional mild one, which usually could be attributed to some definite fault.

The meningococcus antigen, as now used, is prepared by growing meningococci for 18 to 24 hours at $37 \mathrm{C}$. on glucose serum agar in large test tubes. About 35 strains, embracing the various types of meningococci, are used. Approximately $10 \mathrm{cc}$ of normal salt solution are added to each of half of the cultures and the growth washed off; this suspension is then added, 8 or $10 \mathrm{cc}$ to each remaining tube and the organisms suspended in the fluid. It is then centrifuged for about 1 hour, or until the bacteria are closely packed and the supernatant fluid entirely removed, the bacteria are resuspended in salt solution, strained through sterile, fine meshed gauze, and by comparison with a standard turbidity scale the number of bacteria are adjusted to approximately 5,000 million per $c c$. The antigen thus prepared is injected immediately, using a small needle and making the injection slowly. The volume injected never exceeds $25 \mathrm{cc}$. When dead cultures are to be used, the bacteria are killed after the emulsion has been strained. The meningococci and pneumococci are killed by heating at 53 to $55 \mathrm{C}$. for 30 minutes.

Antigens from pneumococci are prepared by inoculating 2 liter bottles of dextrose broth, with a culture which has been passed through mice each week, growing at $37 \mathrm{C}$. for 16 to 20 hours, and the bacteria collected from this broth culture by passing through a Sharples' centrifuge. The pneumococci are suspended in salt solution, centrifuged, the supernatant fluid removed, washed once and a standard suspension of 5,000 million per $\mathrm{cc}$ made. For other bacterial antigens the same procedure is followed. 
The points to be noted carefully are: young cultures, washing the suspension of bacteria, careful straining of the emulsion to remove large clumps of bacteria and particles of agar, prompt injection after washing, and slow injection by means of a small needle.

Most of those who have made use of the intravenous method of inoculation for the production of antimeningococcic serum have begun with the injection of very small amounts of antigen. Amoss and Wollstein used $0.1 \mathrm{cc}$ of an agar slant suspended in $15 \mathrm{cc}$ of salt solution: this was equivalent to about $1 / 20$ of an agar slant. We had begun with $0.1 \mathrm{cc}$ of a polyvalent antigen and increased the amount $0.1 \mathrm{cc}$ at each injection. We found this method would in time cause the development of antibodies in satisfactory amounts, but sought for a plan by which the immunization could be more rapidly brought about, and found that by the use of killed cultures this end could be attained. The scheme of treatment finally found to give the quickest and most satisfactory results was to begin with $0.5 \mathrm{cc}$ of a killed washed polyvalent suspension of meningococci; the amount was increased $0.25 \mathrm{c} c$ each day for 4 days with a resting period of 3 days, when the treatment was resumed, starting with the amount given at the last injection of the previous series. In the second series the dose was increased $0.5 \mathrm{c} c$ and in subsequent series $1 \mathrm{c} c$ at each dose. It was usually found that after the fourth series the antibodies were present in measurable amounts. After the fourth series of washed killed meningococci, the injection of live organisms was begun, the initial dose of washed live bacteria being $1 \mathrm{cc}$ of an approximately 5,000 million per $\mathrm{c} c$ emulsion. Four daily injections were given with a resting period of 3 days between each series. The dose was increased at each injection from 0.5 to $1 \mathrm{cc}$.

A test bleeding was made after the third series of injections of live organisms so that such adjustments might be made in the composition of the antigen as would be most likely to produce a well balanced serum of equal valency to the various groups of meningococci.

For the other antibacterial serums, such as antipneumococcus and antistreptococcus serum, the same scheme was followed with such modifications as were desirable.

We have used the methods described for the immunization of over 200 horses and have not lost a single horse from acute death following the injection, nor have we had what might be called a severe reaction in any horse. Horses have died from the development of cardiac lesions, particularly lesions of the heart valves, but we 
have not lost a horse used in the production of antimeningococcus serum for more than two years. Furthermore, these horses remain in good condition and the meningococcus horses are remarked as being in the best condition of all the horses used for the production of biologic products.

The results obtained in two groups of horses, one against meningococci and one against pneumococci, are given in order that the value of the plan as outlined may be the better appreciated.

In the meningococcus group there were 18 horses, whose treatment was begun at approximately the same time. The average period required before these horses produced a serum which would pass the requirements of the Hygienic Laboratory was 50.1 days; the shortest time for any of the groups was 44 days and the longest was 79. None of these horses had a severe reaction and of course none died from an injection.

In the pneumococcus group there were 49 horses whose treatment was begun about the same time. The average time required to immunize these horses to produce a serum to pass the requirements of the Hygienic Laboratory was 46 days; the shortest period was 34 days, of which there were not less than 7 in the group; the longest was 56 days.

It is quite possible that if all of the horses in both groups had been tested earlier that a number would have been ready for production before the fact was actually determined; but the showing is sufficient to demonstrate the great saving of the method in time and animals.

During the period of greatest demand for antimeningococcic serum, it became important that the interval between bleedings be reduced to the shortest time possible consistent with the maintenance of a high titer for the serum and good condition of the horses. We found that after the horses had reached the production point, there was no difficulty in maintaining them so, and even in increasing the titer of their serum by a single course of injections of antigen between bleedings. In this way we were able to bleed the horses for a time on an average of once in 16 days. We were able to increase the yield of serum by taking 2 bleedings, each of $6,000 \mathrm{cc}, 48$ hours apart, the first bleeding was taken 6 days after the last. injection. By this means the percentage yield of serum to total amount of blood drawn was much increased over that from a single large bleeding. The 
horses stood this intensive treatment well and at the end, without exception, the titer of the serum of all horses had increased.

It is now over two years since some of these horses were started on the production of antimeningococcic serum, and they are in good condition and producing a well balanced serum of a titer much higher than that required by the U. S. Public Health Service.

\section{SUMMARY}

A potent and well balanced antimeningococcic serum can be quickly and safely produced from the horse by the use of the method described.

The method involves the use of an antigen, given intravenously, prepared from washed, killed cultures of meningococci.

The severe and even fatal reactions so frequently encountered in the horse after intravenous injections of bacteria are not a manifestation of anaphylaxis, but are due to the presence of a toxic substance in the material injected.

This toxic material can be removed by centrifugation and washing, and its removal apparently does not reduce the antigenic properties of the emulsion.

A reaction similar to that observed in horses can be produced in rabbits by a first injection of an emulsion of unwashed meningococci; this reaction in the rabbit can be avoided by the use of an emulsion of washed meningococci.

The reaction has no relation to the number of the injection in a series, the series of injection, the rate of injection, or whether live or dead organisms are given, but occurs when the injection is intravenous and unwashed cultures are used.

Points to be noted carefully in the preparation of the antigen are: young cultures, washing the bacterial emulsion, straining the emulsion to remove large clumps and particles of mediums, prompt injection after washing, and slow injection.

Horses can be quickly brought to a production point by the use of washed killed cultures followed at a later period by washed live cultures.

The scheme of injection provides for 4 successive injections with a resting period of 3 days. The treatment is begun with killed cultures, changing to live organisms after the fourth series of injections. 
The average time required to immunize satisfactorily a group of 18 horses to meningococci was 50.1 days; the shortest time was 44 days and the longest was 79 days.

The average time required to immunize a group of 49 horses to pnetumococci was 46 days; the shortest was 34 days, and the longest 56 days.

The method is generally applicable to the production of bacterial antiserum.

\section{CONCLUSIONS}

The method reduces the period required to immunize horses for the production of antimeningococcic and other bacterial antiserums.

The severe and fatal general reactions following the intravenous injection of bacterial emulsions are due to a toxic substance in the emulsion and are not anaphylactic reactions.

This toxic substance can be almost entirely removed by washing the bacteria. 